\title{
Zero-Forcing Frequency-Domain Equalization for Generalized DMT Transceivers with Insufficient Guard Interval
}

\author{
Tanja Karp \\ Department of Electrical and Computer Engineering, Texas Tech University, Lubbock, TX 79409, USA \\ Email: tanja.karp@ttu.edu \\ Steffen Trautmann \\ Infineon Technologies Austria AG, Development Center Villach Siemens Strasse 2, 9500 Villach, Austria \\ Email: steffen.trautmann@infineon.com
}

\author{
Norbert J. Fliege \\ Institute of Computer Engineering, Mannheim University, 68131 Mannheim, Germany \\ Email: fliege@informatik.uni-mannheim.de
}

Received 28 February 2003; Revised 16 September 2003

\begin{abstract}
We propose a zero-forcing frequency domain block equalizer for discrete multitone (DMT) systems with a guard interval of insufficient length. In addition to the insufficient guard interval in the time domain, the equalizer takes advantage of frequency domain redundancy in the form of subcarriers that do not transmit any data. After deriving sufficient conditions for zero-forcing equalization, that is, complete removal of intersymbol and intercarrier interference, we calculate the noise enhancement of the equalizer by evaluating the signal-to-noise ratio (SNR) for each subcarrier. The SNRs are used by an adaptive loading algorithm. It decides how many bits are assigned to each subcarrier in order to achieve a maximum data rate at a fixed error probability. We show that redundancy in the time domain can be traded off for redundancy in the frequency domain resulting in a transceiver with a lower system latency time. The derived equalizer matrix is sparse, thus resulting in a low computational complexity.
\end{abstract}

Keywords and phrases: discrete multitone modulation, insufficient guard interval, zero-forcing frequency domain equalization, noise enhancement, system latency time.

\section{INTRODUCTION}

Discrete multitone (DMT) modulation has been standardized for high data rate transmission over twisted-pair copper wires such as in asymmetric digital subscriber lines (ADSL) and very high bitrate digital subscriber lines (VDSL), which allow transmission speeds up to $8 \mathrm{Mbps}$, or $50 \mathrm{Mbps}$, respectively, over ordinary twisted-pair copper lines of distances up to $4 \mathrm{~km}$. The block diagram of a DMT transceiver is shown in Figure 1. In order to achieve easy equalization at the receiver, a guard interval is introduced at the transmitter in form of a cyclic prefix. Its length has to be at least as long as the memory of the channel.

Coupling the guard interval to the length of the channel impulse response has turned out to be a severe limitation of DMT. For twisted-pair copper wires, the length of the impulse response increases with the length of the line. Thus, if the guard interval is fixed to a maximum length, the channel length has to be restricted to a maximum, too, resulting in applications over short distances, as, for example, VDSL. Increasing the guard interval for a fixed block length $M$ reduces the channel throughput, since the guard interval contains redundant samples only. If we increase the block length by the same amount as the guard interval, in order to maintain a reasonable bandwidth efficiency, this also increases the latency time. Note that the latency time is proportional to $M+L$, where $L$ denotes the size of the guard interval, due to the $\mathrm{P} / \mathrm{S}$ and S/P converters in Figure 1 and is a crucial parameter in many applications. Because of a too high latency time, DMT has been rejected in the ANSI standard for HDSL2 [1]. In order to limit the system latency time while keeping the bandwidth efficiency high, transmission with an insufficient guard interval has been proposed, resulting in new receiver concepts. The following equalizer schemes have been proposed in the literature. 


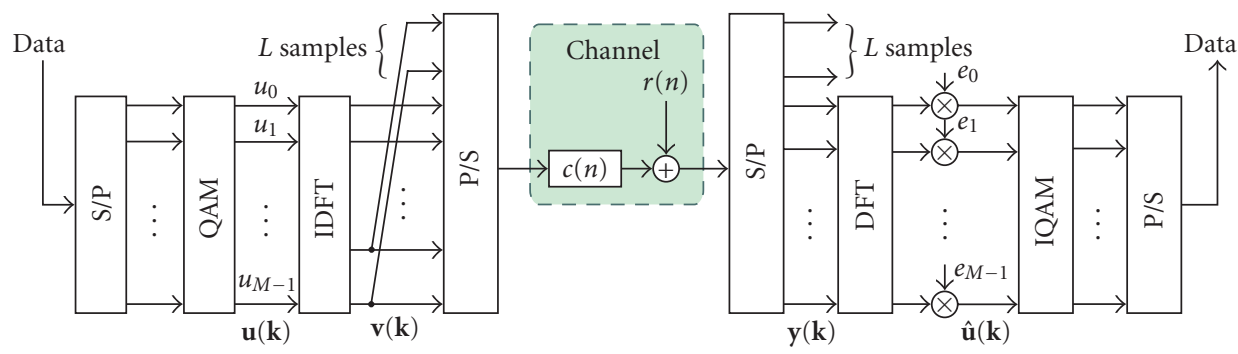

(a)

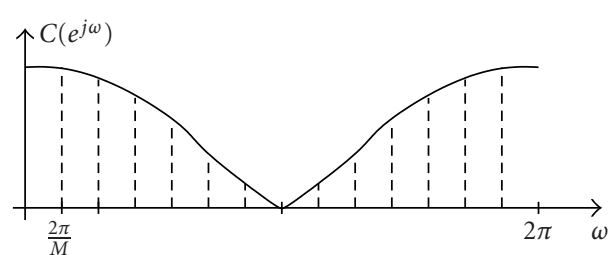

(b)

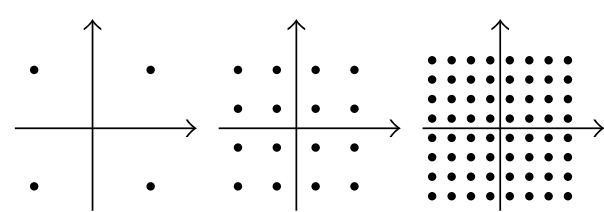

(c)

FIGURE 1: (a) DMT transmission scheme, (b) subchannels of the transmission channel, and (c) different possible QAM schemes.

\section{Time-domain equalizer (TEQ)}

The TEQ is a short FIR filter at the receiver input that is designed to shorten the duration of the channel impulse response. It thus allows a reduction of the guard interval $[2,3,4,5,6]$. Using a filter with up to 20 coefficients, the effective channel impulse response of a typical copper wire can easily be reduced by a factor of 10 . Different cost functions such as minimum mean squared error $[2,4,5]$, maximum shortening signal-to-noise ratio (SNR) [3], maximum geometric SNR [2], minimum intersymbol interference (ISI) [6], and maximum bitrate [6] have been proposed to design the TEQ. An overview of the different methods and their performances is presented in [6]. Only the maximum bitrate method is optimal in terms of achievable bitrate, but its high computational complexity is prohibitive for a practical implementation [6].

\section{Per-tone equalization}

In per-tone equalization [7], the TEQ is transferred to the frequency domain, resulting in a complex frequency domain equalizer for each tone. This allows to optimize the SNR and therewith the bitrate for each tone individually. Furthermore, the equalization effort can be concentrated on the most affected tones by increasing the number of equalization filter coefficients for these tones. No effort is wasted to equalize unused subcarriers when setting the number of taps for their equalizers to zero. However, the computational complexity of the algorithm is still relatively high.

\section{Multiple-input multiple-output (MIMO) equalization}

The MIMO equalizer replaces either the one-tap equalizer in the DMT receiver or the sequence of guard interval removal,
DFT, and one-tap equalizer by a MIMO FIR or IIR filter $[8,9,10,11,12,13]$. Depending on the cost function applied to optimize the MIMO equalizer, we can distinguish between zero-forcing ( $\mathrm{ZF})$ equalization and minimum mean squared error (MMSE) equalization. ZF equalizers totally eliminate ISI and intercarrier interference (ICI), while MMSE equalizers also include additive channel noise in the cost function. It has been shown in $[8,12]$ that ISI and ICI can be completely removed if the guard interval is at least of length $L=1$ and if the $M+L$ polyphase components $[14,15]$ of the channel impulse response do not have common zeros. A sufficient condition is given for the length of the FIR equalizers, which decreases with an increase of the guard interval $L$. In [12], it has been proved that perfect equalization is even possible for common zeros of the channel polyphase components if redundancy is not introduced in terms of a cyclic prefix but as a trailing block of zeros.

\section{Adaptive signal processing}

In [16], it is proposed to replace the fixed size fast Fourier transform (FFT) in the receiver by a variable length window. A part of the received signal and the ISI is discarded. ICI due to lost orthogonality of the new windowing technique is then removed in a matched filter multistage ICI canceller.

\section{Generalized DMT (GDMT)}

More recently a different frequency domain equalizer has been introduced under the name of GDMT $[17,18]$. Here, the one tap frequency domain equalizer of a traditional DMT receiver is replaced by a block equalizer matrix and the guard interval is omitted. The equalizer takes advantage of inherent frequency domain redundancy in DMT due to unused tones, 
that is, subcarriers to which the adaptive loading algorithm does not assign any data due to a too low SNR. These subcarriers do not need to be equalized at the receiver, but they contain information that can be exploited to obtain a better compensation of ISI and ICI in used subcarriers. Note that the idea of exploiting unused subcarriers is not totally new, but has already been successfully applied to reduce the peakto-average power ratio at the transmitter [19, 20, 21, 22, 23].

We here extend the equalizer concept proposed in GDMT to the case of an existing but insufficient guard interval. The outline of the paper is as follows. In Section 2, the input/output relationship of a DMT transceiver is given in terms of a matrix and vector representation, assuming that the one-tap equalizer per subcarrier in a traditional DMT receiver has been replaced by a block equalizer. We then derive conditions for ZF equalization, that is, perfect removal of ISI and ICI, in Section 3 and show that it cannot be achieved by the proposed block equalizer in the case of a guard interval of insufficient length if all subcarriers are used for data transmission. Section 4 derives the equalizer coefficients if some subcarriers are not used for data transmission and thus do not need to be equalized, as well as a condition on how many unused subcarriers are needed for ZF equalization. The noise variance at the equalizer output is also derived for each subcarrier and compared to the case of a guard interval of sufficient length. Section 5 describes how to exploit the derived subcarrier SNRs in order to assign used and unused subcarriers and to determine the bitload of used subcarriers in an adaptive loading algorithm. Section 6 shows simulation results and compares them to the performance of a TEQ. Section 7 summarizes the results in a conclusion.

\subsection{Notation and definitions}

Bold face letters denote vectors (if lowercase) and matrices (if uppercase). $\mathbf{A}^{T}, \mathbf{A}^{H}$, and $\mathbf{A}^{\dagger}$ denote the transpose, Hermitean, and pseudoinverse of matrix $\mathbf{A}$, respectively. $[A]_{k, l}$ denotes the element in row $k$ and column $l$ of the matrix; $\operatorname{diag}(\mathbf{A})$ converts $\mathbf{A}$ into a diagonal matrix by extracting the diagonal elements, and $\operatorname{diag}(\mathbf{x})$ creates a diagonal matrix from vector $\mathbf{x}$ by placing the vector elements on the diagonal of the matrix. The nullspace of $\mathbf{A}$, denoted as $\mathcal{N}(\mathbf{A})$, is defined by the set of vectors $\mathbf{x}$ such that $\mathbf{A x}=\mathbf{0}$ and the left nullspace of $\mathbf{A}$ is defined by the set of vectors $\mathbf{y}$ such that $\mathbf{y}^{H} \mathbf{A}=\mathbf{0}$.

\section{THE DMT TRANSCEIVER}

The relationship between the DMT input symbol $\mathbf{u}(k)$ and the output symbol $\hat{\mathbf{u}}(k)$ in Figure 1 is given by $[8,12,24]$

$$
\begin{aligned}
\hat{\mathbf{u}}(k) & =\mathbf{E} \frac{\mathbf{W}_{M}}{\sqrt{M}} \mathbf{Z}_{R} \\
& \cdot\left(\left[\begin{array}{ll}
\mathbf{C}_{0} & \mathbf{C}_{1}
\end{array}\right]\left[\begin{array}{cc}
\mathbf{Z}_{T} & \mathbf{0} \\
\mathbf{0} & \mathbf{Z}_{T}
\end{array}\right]\left[\begin{array}{cc}
\frac{\mathbf{W}_{M}^{H}}{\sqrt{M}} & \mathbf{0} \\
\mathbf{0} & \frac{\mathbf{W}_{M}^{H}}{\sqrt{M}}
\end{array}\right]\left[\begin{array}{c}
\mathbf{u}(k-1) \\
\mathbf{u}(k)
\end{array}\right]+\mathbf{r}(k)\right),
\end{aligned}
$$

where $\mathbf{E}=\operatorname{diag}\left(\left[e_{0}, \ldots, e_{M-1}\right]\right)$ denotes the one-tap equalizer per subcarrier. $\mathbf{W}_{M} / \sqrt{M}$ and $\mathbf{W}_{M}^{H} / \sqrt{M}$ describe the orthonormal DFT and IDFT matrix, respectively, and $\mathbf{Z}_{T}$ and $\mathbf{Z}_{R}$ the introduction and removal of the guard interval of size $L$, respectively. $\mathbf{C}=\left[\begin{array}{ll}\mathbf{C}_{0} & \mathbf{C}_{1}\end{array}\right]$ is the size $(M+L) \times 2(M+L)$ channel matrix combining the $\mathrm{P} / \mathrm{S}$ conversion at the transmitter, the convolution with the channel impulse response and the $\mathrm{S} / \mathrm{P}$ conversion at the receiver, and $\mathbf{r}(k)$ is the additive channel noise after S/P conversion. We here assume that the channel impulse response $c(n)$ is of length $L_{c}$ and shorter than $M$, what is generally the case for ADSL and VDSL. The entries of the matrices are then given by

$$
\begin{gathered}
{\left[\mathbf{W}_{M}\right]_{k, l}=\exp \left(-j \frac{2 \pi k l}{M}\right), \quad\left[\mathbf{W}_{M}^{H}\right]_{k, l}=\exp \left(j \frac{2 \pi k l}{M}\right),} \\
k, l=0, \ldots, M-1, \\
\mathbf{Z}_{T}=\left[\begin{array}{c}
\mathbf{0}_{L \times(M-L)} \mathbf{I}_{L} \\
\mathbf{I}_{M}
\end{array}\right], \quad \mathbf{Z}_{R}=\left[\begin{array}{llll}
\mathbf{0}_{M \times L} & \mathbf{I}_{M}
\end{array}\right], \\
\mathbf{C}_{0}=\left[\begin{array}{ccccc}
0 & \cdots & c_{L_{c}-1} & \cdots & c_{1} \\
\vdots & \ddots & & \ddots & \vdots \\
\vdots & & \ddots & & c_{L_{c}-1} \\
\vdots & & & \ddots & \vdots \\
0 & \cdots & \cdots & \cdots & 0
\end{array}\right], \\
\mathbf{C}_{1}=\left[\begin{array}{ccccc}
c_{0} & 0 & \cdots & \cdots & 0 \\
\vdots & \ddots & \ddots & & \vdots \\
c_{L_{c}-1} & \cdots & c_{0} & \ddots & \vdots \\
& \ddots & & \ddots & 0 \\
0 & & c_{L_{c}-1} & \cdots & c_{0}
\end{array}\right] .
\end{gathered}
$$

The capacity of each subcarrier depends on the subcarrier output SNR and is given by $C_{k}=\log _{2}\left(1+\mathrm{SNR}_{k}\right)$ [25]. The actual bitload per subcarrier is then given by

$$
b_{k}=\log _{2}\left(1+\frac{\mathrm{SNR}_{k}}{\Gamma}\right) \text {, }
$$

where $\Gamma$ is called the SNR gap and depends on the target bit error rate, the modulation scheme, and whether channel coding is performed, see [25] for details. The division of the channel frequency response into $M$ subchannels as well as different QAM constellations are demonstrated in Figure 1. The bitrate per DMT symbol is then calculated as $B=\sum_{k=0}^{M / 2} b_{k}$, where the summation index $k$ only runs to $M / 2$ since the subcarriers $M / 2+1$ to $M-1$ carry complex conjugate QAM symbols of the subcarriers $M / 2-1$ to 1 in order to assure a real-valued transmit signal. Integer solutions for (3) that maximize the bitrate per DMT symbol given the SNR per subchannel, the target SNR gap, and a maximally allowable transmit power are found through an adaptive loading 


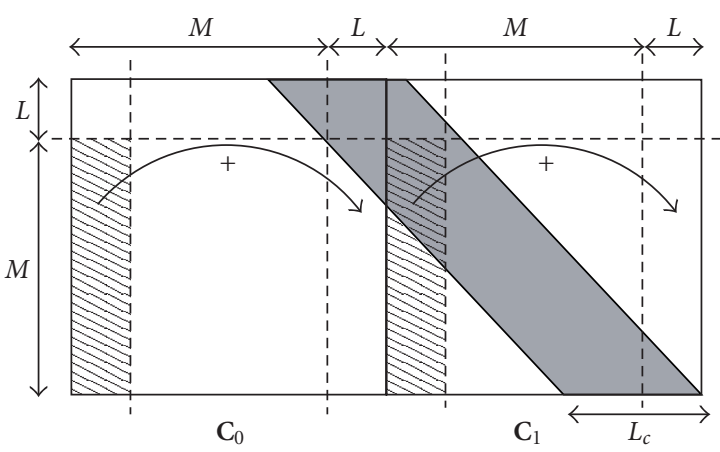

FIGURE 2: Influence of introducing and removing the guard interval on the channel matrix.

algorithm [26, 27, 28, 29, 30, 31, 32] the transmitter. These values are used to initialize the QAM modulator in Figure 1. Subchannels with a low SNR might end up not to carry any data since it turns out to be more favorable to spend the transmit energy of these subcarriers to increase the bitload on subcarriers with a high SNR. Since for twisted-pair copper wires the transmission channel can be considered as time invariant, the initialization has to be performed only once and is based on an estimate of the channel frequency response.

\section{ZERO FORCING EQUALIZATION}

A ZF equalizer removes the ISI and ICI introduced by the transmission channel. It is designed for the noise-free case and does not take noise enhancement into consideration. We at this point remove the restriction that the equalizer matrix $\mathrm{E}$ is diagonal, but we assume a general $M \times M$ matrix. Starting from (1), ISI and ICI are removed if the following condition holds true:

$$
\hat{\mathbf{u}}(k)=\underbrace{\left[\begin{array}{ll}
\mathbf{0}_{M} & \mathbf{I}_{M}
\end{array}\right]\left[\begin{array}{c}
\mathbf{u}(k-1) \\
\mathbf{u}(k)
\end{array}\right]}_{\mathbf{u}(k)}+\mathbf{E} \frac{\mathbf{W}_{M}}{\sqrt{M}} \mathbf{Z}_{R} \mathbf{r}(k),
$$

or equivalently,

$$
\frac{1}{M} \mathbf{E W}_{M} \mathbf{Z}_{R}\left[\mathbf{C}_{0} \mathbf{C}_{1}\right]\left[\begin{array}{cc}
\mathbf{Z}_{T} & \mathbf{0} \\
\mathbf{0} & \mathbf{Z}_{T}
\end{array}\right]\left[\begin{array}{cc}
\mathbf{W}_{M}^{H} & \mathbf{0} \\
\mathbf{0} & \mathbf{W}_{M}^{H}
\end{array}\right]=\left[\begin{array}{ll}
\mathbf{0}_{M} & \mathbf{I}_{M}
\end{array}\right]
$$

To find the entries of $\mathbf{E}$, we first consider the influence on the channel matrix $\mathbf{C}$ of introducing and removing the guard interval, see Figure 2. The gray diagonal band in the matrix in Figure 2 denotes the nonzero entries. The introduction of the guard interval causes the first $L$ columns of $\mathbf{C}_{0}$ and $\mathbf{C}_{1}$, respectively, to be moved and added to their last $L$ columns. Then the removal of the guard interval reduces the matrices by its first $L$ rows. We call the matrix resulting from these

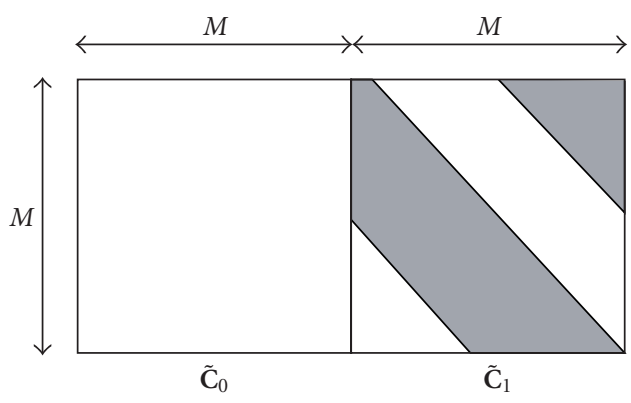

Figure 3: $\tilde{\mathbf{C}}=\left[\begin{array}{ll}\tilde{\mathbf{C}}_{0} & \tilde{\mathbf{C}}_{1}\end{array}\right]$ for a guard interval of sufficient length.

operations $\tilde{\mathbf{C}}$ with

$$
\tilde{\mathbf{C}}=\left[\begin{array}{ll}
\tilde{\mathbf{C}}_{0} & \tilde{\mathbf{C}}_{1}
\end{array}\right]=\mathbf{Z}_{R}\left[\begin{array}{ll}
\mathbf{C}_{0} & \mathbf{C}_{1}
\end{array}\right]\left[\begin{array}{cc}
\mathbf{Z}_{T} & \mathbf{0} \\
\mathbf{0} & \mathbf{Z}_{T}
\end{array}\right] .
$$

Introducing $\tilde{\mathbf{C}}$ into (5) and splitting it into two parts, we obtain as constraints for ZF equalization the following two equations:

$$
\begin{aligned}
\frac{\mathbf{E} \mathbf{W}_{M} \tilde{\mathbf{C}}_{0} \mathbf{W}_{M}^{H}}{M} & =\mathbf{0}_{M}, \\
\frac{\mathbf{E W}_{M} \tilde{\mathbf{C}}_{1} \mathbf{W}_{M}^{H}}{M} & =\mathbf{I}_{M} .
\end{aligned}
$$

\subsection{Guard interval of sufficient length}

If the guard interval is of sufficient length, that is, $L \geq L_{c}-$ 1, then $\tilde{\mathbf{C}}=\left[\begin{array}{ll}\tilde{\mathbf{C}}_{0} & \tilde{\mathbf{C}}_{1}\end{array}\right]$ has the following structure, see also Figure 3:

$$
\begin{aligned}
\tilde{\mathbf{C}}_{0} & =\mathbf{0}_{M}, \\
\tilde{\mathbf{C}}_{1} & =\left[\begin{array}{cccccc}
c_{0} & & & c_{L_{c}-1} & \cdots & c_{1} \\
\vdots & \ddots & & & \ddots & \vdots \\
\vdots & & \ddots & & & c_{L_{c}-1} \\
c_{L_{c}-1} & & & \ddots & & \\
& \ddots & & & \ddots & \\
& & c_{L_{c}-1} & \cdots & \cdots & c_{0}
\end{array}\right] .
\end{aligned}
$$

Thus, in this case (7) is always satisfied since $\tilde{\mathbf{C}}_{0}=\mathbf{0}_{M}$, and since $\tilde{\mathbf{C}}_{1}$ is circular, $\mathbf{W}_{M} \tilde{\mathbf{C}}_{1} \mathbf{W}_{M}^{H} / M$ in (8) becomes a diagonal matrix $\mathbf{D}$ whose entries are given by the $M$-point DFT of the channel impulse response. The ZF equalizer $\mathbf{E}$ is identical to the DMT equalizer, namely,

$$
\mathbf{E}=\mathbf{D}^{-1}, \quad[\mathbf{E}]_{k, k}=\frac{1}{C\left(e^{j 2 \pi k / M}\right)}, \quad k=0, \ldots, M-1,
$$


where $C\left(e^{j 2 \pi k / M}\right)$ denotes the channel frequency response at the normalized frequencies $2 \pi k / M$. Note that the equalizer coefficients are only defined as long as the channel does not have a spectral null at one of the subcarrier frequencies. If the latter is the case, the subcarrier cannot be used for data transmission and the adaptive loading algorithm at the transmitter would decide not to transmit any data through that particular subchannel. Thus there is no need to equalize that subchannel. For an arbitrary channel frequency response, the equalizer coefficients can be described as

$$
\mathbf{E}=\mathbf{C}_{\text {freq }}^{\dagger}
$$

with

$$
\mathrm{C}_{\text {freq }}=\operatorname{diag}\left(\left[C\left(e^{j 0}\right), C\left(e^{j 2 \pi 1 / M}\right), \ldots, C\left(e^{j 2 \pi(M-1) / M}\right)\right]\right)
$$

and $\mathbf{C}_{\text {freq }}^{\dagger}$ being its also diagonal pseudoinverse,

$$
\begin{aligned}
{\left[\mathbf{C}_{\text {freq }}^{\dagger}\right]_{k, k}=} & \begin{array}{ll}
\frac{1}{C\left(e^{j 2 \pi k / M}\right)}, & \text { if } C\left(e^{j 2 \pi k / M}\right) \neq 0, \\
0, & \text { else, }
\end{array} \\
& k=0, \ldots, M-1 .
\end{aligned}
$$

One critical aspect of a ZF equalizer is the noise enhancement it may result in. Given the variance $\sigma_{r}^{2}$ of the additive channel noise, we can calculate the noise variance $\sigma_{n, k}^{2}$ at the output of subband $k$ as

$$
\begin{aligned}
\operatorname{diag} & \left(\left[\sigma_{n, 0}^{2}, \sigma_{n, 1}^{2}, \ldots, \sigma_{n, M-1}^{2}\right]\right) \\
& =\sigma_{r}^{2} \cdot \operatorname{diag}\left(\mathbf{E} \frac{\mathbf{W}_{M}}{\sqrt{M}} \cdot \frac{\mathbf{W}_{M}^{H}}{\sqrt{M}} \mathbf{E}^{H}\right) \\
& =\sigma_{r}^{2} \cdot \operatorname{diag}\left(\mathbf{E} \cdot \mathbf{E}^{H}\right) \\
& =\sigma_{r}^{2} \mathbf{C}_{\text {freq }}^{\dagger}\left(\mathbf{C}_{\text {freq }}^{\dagger}\right)^{H} .
\end{aligned}
$$

The noise variance in a subcarrier is proportional to the inverse of the squared channel magnitude response at the subcarrier frequency, that is, it is low in "good" subcarriers and high in "bad" subcarriers. Since all ISI and ICI have been removed by the equalizer the SNR in subcarrier $k$ is given by

$$
\mathrm{SNR}_{k}=10 \log _{10} \frac{\sigma_{u_{k}}^{2}\left|C\left(e^{j 2 \pi k / M}\right)\right|^{2}}{\sigma_{r}^{2}}, \quad k=0, \ldots, M-1,
$$

where $\sigma_{u_{k}}^{2}$ describes the variance of the QAM signal transmitted in subcarrier $k$.

\subsection{Guard interval of insufficient length}

If however the guard interval is of insufficient length, that is, $L<L_{c}-1$, then $\tilde{\mathbf{C}}_{0}$ and $\tilde{\mathbf{C}}_{1}$ have the following form (see also

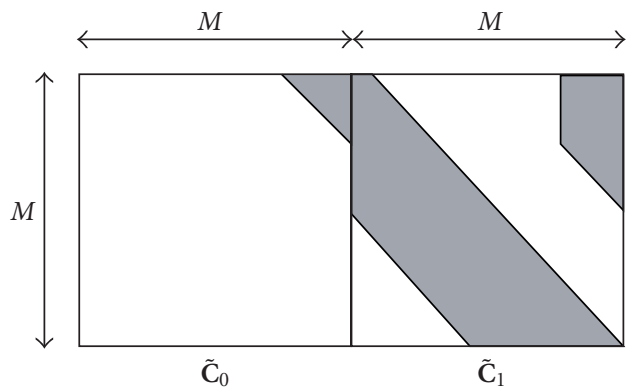

FIgURE 4: $\tilde{\mathbf{C}}=\left[\begin{array}{ll}\tilde{\mathbf{C}}_{0} & \tilde{\mathbf{C}}_{1}\end{array}\right]$ for a guard interval of insufficient length.

Figure 4):

$$
\begin{aligned}
& \tilde{\mathbf{C}}_{0}=\left[\begin{array}{cccccc}
0 & \cdots & 0 & c_{L_{c}-1} & \cdots & c_{L+1} \\
& & & & \ddots & \vdots \\
\vdots & & & & & c_{L_{c}-1} \\
& & & & & 0 \\
& & & & & \vdots \\
0 & & & \cdots & & 0
\end{array}\right]
\end{aligned}
$$

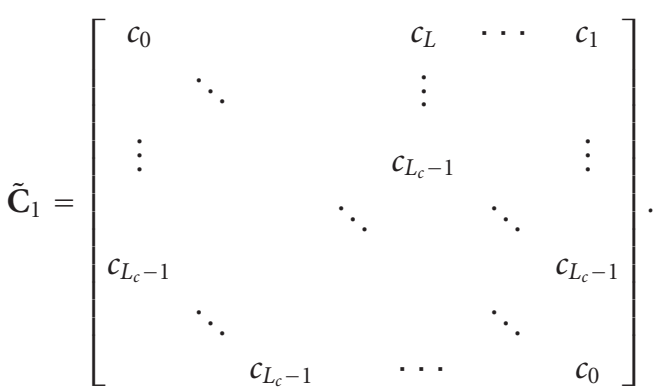

In order to satisfy (7), we now need to ensure that $\left.(\mathbf{E W})_{M}\right)^{H}$ lies in the left nullspace of $\tilde{\mathbf{C}}_{0}$. Since the rank of $\tilde{\mathbf{C}}_{0}$ is $L_{c}-L-1$, the dimension of the left nullspace is $M-L_{c}+L+1$. Thus $\left(\mathbf{E W}_{M}\right)^{H}=\mathbf{W}_{M}^{H} \mathbf{E}^{H}$ contains $M-L_{c}+L+1$ nontrivial linear independent column vectors and since $\mathbf{W}_{M}^{H}$ is an orthogonal transform, the same holds true for $\mathbf{E}^{H}$. Since $\tilde{\mathbf{C}}_{0}$ is an upper triangular matrix, we also know that $\mathbf{E W}_{M}$ must have the following form to meet the nullspace condition:

$$
\mathbf{E W}_{M}=\left[\begin{array}{ll}
\mathbf{0}_{M \times L_{c}-L-1} & \mathbf{X}_{M \times M-L_{c}+L+1}
\end{array}\right],
$$

where $\mathbf{X}_{M \times M-L_{c}+L+1}$ denotes a matrix of don't care entries. We assume that the nullspace constraint is satisfied. Then, instead of solving (8) directly, we can also solve for

$$
\mathbf{E W}_{M} \underbrace{\left(\tilde{\mathbf{C}}_{1}+\tilde{\mathbf{C}}_{0} \mathbf{P}\right)}_{\tilde{\mathbf{C}}_{\text {circ }}} \frac{\mathbf{W}_{M}^{H}}{M}=\mathbf{I}_{M}
$$

with

$$
\mathbf{P}=\left[\begin{array}{cc}
\mathbf{0}_{L \times(M-L)} & \mathbf{I}_{L} \\
\mathbf{I}_{M-L} & \mathbf{0}_{(M-L) \times L}
\end{array}\right]
$$




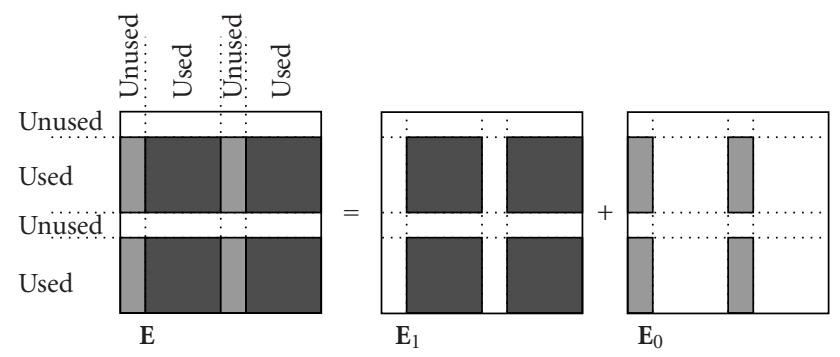

FIgURe 5: Decomposition of equalizer matrix. White rows and columns denote zero entries.

since we have only added a zero matrix to the left- and righthand sides of (8). The matrix $\mathbf{P}$ shifts the entries of $\tilde{\mathbf{C}}_{0}$ by $L$ columns to the left such that $\tilde{\mathbf{C}}_{\text {circ }}=\tilde{\mathbf{C}}_{1}+\tilde{\mathbf{C}}_{0} \mathbf{P}$ is again a circular matrix that is diagonalized through multiplication with the DFT and IDFT matrices and results in (11) as a solution for (19). However, it can be easily verified that this solution does not satisfy the nullspace constraint in (18) and therefore it is impossible to solve (7) and (8) simultaneously.

\section{ZERO-FORCING EQUALIZATION FOR TRANSMISSION WITH INSUFFICIENT GUARD INTERVAL AND UNUSED SUBCARRIERS}

We now assume that $K$ subcarriers are not used for data transmission, that is, the value zero is transmitted in these subcarriers, and that the guard interval is of insufficient length. Note that in DMT transceivers there are generally some subcarriers that are not assigned any data by the adaptive loading algorithm. The block equalizer $\mathbf{E}$ then only needs to equalize the $N=M-K$ subcarriers used for data transmission, since there is no need to equalize unused subcarriers. In particular, this means that (19) only has to be satisfied for the used subcarriers. Since $\mathbf{W}_{M} \tilde{\mathbf{C}}_{\text {circ }} \mathbf{W}_{M}^{H} / M$ is diagonal, all rows and columns of $\mathbf{E}$ according to (11) corresponding to unused subcarriers can be chosen arbitrarily and (19) is still satisfied for all used subcarriers.

In the following, we split $\mathbf{E}$ into a sum of two matrices, $\mathbf{E}_{0}$ and $\mathbf{E}_{1}$, where $\mathbf{E}_{1}$ describes a particular solution of (19), where all the arbitrary entries are chosen to be zeros and $\mathbf{E}_{0}$ describes the arbitrary entries. The structures of $\mathbf{E}_{1}$ and $\mathbf{E}_{0}$ are shown in Figure 5. Note that even for $\mathbf{E}_{0}$ we have chosen the rows corresponding to unused subcarriers equal to zero. The entries in these rows are not needed since they only describe the equalizer output in the unused subcarriers.

$E_{1}$ can then be obtained from solving (19) for the used subcarriers only. The solution is identical to that part of (11) that corresponds to the used subcarriers and can mathematically be described by

$$
\mathbf{E}_{1}=\mathbf{S}_{1} \mathbf{C}_{\text {freq }}^{\dagger}
$$

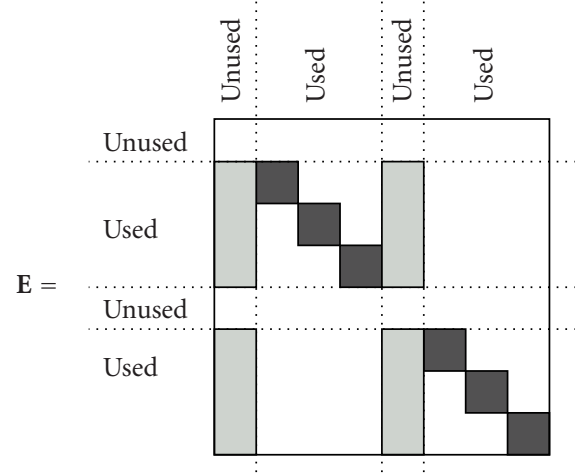

FIGURE 6: Nonzero entries of equalizer matrix.

where $\boldsymbol{S}_{1}$ denotes a carrier selection matrix

$$
\begin{gathered}
\boldsymbol{S}_{1}=\operatorname{diag}\left(s_{0}, \ldots, s_{M-1}\right), \\
s_{i}= \begin{cases}1, & \text { subcarrier is used, } \\
0, & \text { subcarrier is unused, }\end{cases}
\end{gathered}
$$

and ensures that $\mathbf{E}_{1}$ has zero entries in rows and columns corresponding to unused subcarriers. We can now use $\mathbf{E}_{0}$ to satisfy the nullspace constraint in (18). An equivalent way to express (18) without including the don't care matrix $\mathbf{X}$ is given by

$$
\underbrace{\mathbf{E} \mathbf{W}_{M}\left[\begin{array}{c}
\mathbf{I}_{L_{c}-L-1} \\
\mathbf{0}_{\left(M-\left(L_{c}-L-1\right)\right) \times\left(L_{c}-L-1\right)}
\end{array}\right]}_{\mathbf{W}_{0}}=\mathbf{0}_{M \times\left(L_{c}-L-1\right)} .
$$

Note that $\mathbf{W}_{0}$ contains the first $L_{c}-L-1$ of the DFT matrix $\mathbf{W}_{M}$ and thus has full column rank. The only free parameters available to solve (23) are the $K$ columns of nonzero entries in $\mathbf{E}_{0}$ and thus a solution of the linear system of equations exists, if $K \geq L_{c}-L-1$. This means that for each tap that the guard interval is too short, we need one unused subcarrier in order to design an equalizer $\mathbf{E}$ that completely removes ISI and ICI. Replacing $\mathbf{E}$ in (23) by $\mathbf{E}_{0}+\mathbf{E}_{1}$ and introducing an additional matrix $\left(\mathbf{I}_{M}-\mathbf{S}_{1}\right)$ that ensures that $\mathbf{E}_{0}$ has nonzero entries only at columns corresponding to unused subcarriers, we obtain

$$
\begin{gathered}
\mathbf{E}_{0}\left(\mathbf{I}_{M}-\mathbf{S}_{1}\right) \mathbf{W}_{0}=-\mathbf{E}_{1} \mathbf{W}_{0}, \\
\mathbf{E}_{0}=-\mathbf{E}_{1} \mathbf{W}_{0}\left(\left(\mathbf{I}_{M}-\mathbf{S}_{1}\right) \mathbf{W}_{0}\right)^{\dagger} \\
=-\mathbf{S}_{1} \mathbf{C}_{\text {freq }}^{\dagger} \mathbf{W}_{0}\left(\left(\mathbf{I}_{M}-\mathbf{S}_{1}\right) \mathbf{W}_{0}\right)^{\dagger} .
\end{gathered}
$$

Using these results, the equalizer matrix is given as

$$
\mathbf{E}=\mathbf{E}_{0}+\mathbf{E}_{1}=\mathbf{S}_{1} \mathbf{C}_{\text {freq }}^{\dagger}\left(\mathbf{I}_{M}-\mathbf{W}_{0}\left(\left(\mathbf{I}_{M}-\mathbf{S}_{1}\right) \mathbf{W}_{0}\right)^{\dagger}\right) .
$$

The nonzero entries of $\mathbf{E}$ are illustrated in Figure 6. To equalize a used subcarrier, the signal is multiplied with the same scaling factor, determined by the inverse frequency response of the channel at the subcarrier frequency, as in the 
original DMT scheme. In addition, a linear combination of the outputs of all unused subcarriers is added. Note that the values that are received in the unused subcarriers describe ISI and ICI from used subcarriers as well as additive channel noise. The fact that the ISI and ICI component is not negligible is due to the low stopband attenuation of the IDFT at the transmitter that allows significant leakage into neighboring subcarriers. This fact is generally considered as a drawback of using the IDFT and DFT for modulation and demodulation, respectively, but has been exploited as an advantage here. Thanks to its sparse structure, the implementation cost of the ZF block equalizer is low.

Given the equalizer coefficients and the variance $\sigma_{r}^{2}$ of the additive channel noise, we can now calculate the noise variance at the output of the equalizer:

$$
\begin{aligned}
\operatorname{diag} & \left(\left[\sigma_{n, 0}^{2}, \sigma_{n, 1}^{2}, \ldots, \sigma_{n, M-1}^{2}\right]\right) \\
= & \sigma_{r}^{2} \cdot \operatorname{diag}\left(\mathbf{E} \cdot \mathbf{E}^{H}\right) \\
= & \sigma_{r}^{2} \cdot \operatorname{diag}\left(\left(\mathbf{E}_{0}+\mathbf{E}_{1}\right)\left(\mathbf{E}_{0}+\mathbf{E}_{1}\right)^{H}\right) \\
= & \sigma_{r}^{2} \cdot \operatorname{diag}\left(\mathbf{E}_{1} \mathbf{E}_{1}^{H}\right)+\operatorname{diag}\left(\mathbf{E}_{0} \mathbf{E}_{0}^{H}\right) \\
= & \sigma_{r}^{2} \mathbf{C}_{\text {freq }}^{\dagger}\left(\mathbf{C}_{\text {freq }}^{\dagger}\right)^{H} \mathbf{S}_{1} \\
& \cdot\left(\mathbf{I}_{M}+\operatorname{diag}\left(\mathbf{W}_{0}\left(\mathbf{W}_{0}^{H}\left(\mathbf{I}_{M}-\mathbf{S}_{1}\right) \mathbf{W}_{0}\right)^{-1} \mathbf{W}_{0}^{H}\right)\right),
\end{aligned}
$$

where we have taken into account that the products $\mathbf{E}_{0} \mathbf{E}_{1}^{H}$ and $\mathbf{E}_{1} \mathbf{E}_{0}^{H}$ have zero diagonal elements as can be easily verified from Figure 5. The derivation of (31) from (30) is described in Appendix A. Note that the first noise term, that is, $\sigma_{r}^{2} \cdot \operatorname{diag}\left(\mathbf{E}_{1} \mathbf{E}_{1}^{H}\right)$, is the same as in a conventional DMT receiver with diagonal entries only, see (15). The second term arises from the nonzero entries in $\mathbf{E}_{0}$. It is also proportional to the inverse of the squared channel magnitude response at the subcarrier frequency, but in addition depends on the position of the used and unused carriers since it contains the carrier selection matrix $\mathbf{S}_{1}$ inside the inverse matrix.

\section{UNUSED SUBCARRIER SELECTION}

As described in Section 2, the bitload per subcarrier in a DMT transceiver is determined by an adaptive loading algorithm that maximizes the bitrate per DMT symbol given the SNRs per subchannel, a target SNR gap $\Gamma$, and a given maximum transmit power. If the guard interval is of sufficient length, the SNRs per subcarrier are independent of each other, see (16), and the adaptive loading algorithm $[26,27,28,29,30,31,32]$ can iteratively assign bits to the subcarriers starting with the ones having the highest SNR. In the case of an insufficient guard interval, however, we have seen from (31) that the noise enhancement in the receiver not only depends on the channel frequency response but also on the position of the used and unused subcarriers. Therefore, deciding which subcarriers to use becomes a more elaborate task than just assigning the $K$ subcarriers with the highest channel attenuation as the unused ones. In the following, we will look at two special cases first before evaluating the general case.

\subsection{Guard interval is too short by one tap}

If the guard interval is just one tap too short, that is, $L_{c}-L-$ $1=1$, then the matrix $\mathbf{W}_{0}$ in (31) just consists of the first column of $\mathbf{W}_{M}$ and the inverse matrix in (31) is a scalar,

$$
\left(\mathbf{W}_{0}^{H}\left(\mathbf{I}_{M}-\mathbf{S}_{1}\right) \mathbf{W}_{0}\right)^{-1}=\left(\sum_{k=0}^{M-1}\left(1-s_{k}\right)\right)^{-1}=\frac{1}{K}
$$

with $s_{k}$ from (22). Substituting this result into (31), we obtain for the noise variance $\sigma_{n, k}^{2}$ and for the SNR at the output of a used subcarrier $k$,

$$
\begin{aligned}
\sigma_{n, k}^{2} & =\frac{\sigma_{r}^{2}}{\left|C\left(e^{j(2 \pi k / M)}\right)\right|^{2}}\left(1+\frac{1}{K}\right), \\
\mathrm{SNR}_{k} & =\frac{\sigma_{u, k}^{2}}{\sigma_{n, k}^{2}}=\frac{\sigma_{u, k}^{2} \cdot\left|C\left(e^{j(2 \pi k / M)}\right)\right|^{2}}{\sigma_{r}^{2}(1+1 / K)} .
\end{aligned}
$$

In this special case, the SNRs in used subcarriers only depend on the channel magnitude frequency response and the number of unused subcarriers. Thus, once $K$ has been chosen (and it has to be at least one since otherwise ZF equalization is impossible) in order to select the subcarriers resulting in the highest data rate, we just have to choose those $N=M-K$ ones with the highest SNRs.

Note that for $K=1$ the noise variance is twice as high as in DMT with sufficient guard interval, but it reduces as $K$ increases. The optimal value for $K$ can be determined iteratively by starting with $K=1$ and increasing $K$ in steps of one until the data rate stops increasing. At each step, we can apply one of the existing adaptive loading algorithms in order to determine the data rate. The only minor modification that has to be made is to prevent the adaptive loading algorithm from assigning bits to subcarriers declared as unused, for example, by setting the SNR in unused subcarriers to 0 . For each used subcarrier that we convert into an unused one in an iteration step, the noise variance in the remaining used subcarriers reduces. In addition, we can increase the signal variance $\sigma_{u, k}^{2}$ in the used subcarriers, since the total signal power now has to be split over a smaller number of subcarriers. Both effects increase the used subcarriers' SNRs. As long as this improvement allows us to increase the bitload by more bits than the subcarrier we removed was carrying, the total data rate increases. As the iteration continues, the improvement of SNRs in used subcarriers reduces and the SNR of the subcarrier that is converted from used to unused has an increasing SNR. Therefore, at some point, the total bit rate stops increasing and we have found the optimal value for $K$.

\subsection{Unused subcarriers are spaced equidistantly}

The other special case that is easy to solve is where the inverse matrix in (31) is a scaled identity matrix. Remember that $\mathbf{W}_{0}$ consists of the first $L_{c}-L-1$ columns of the $M$-point DFT matrix $\mathbf{W}_{M}$. Taking advantage of the fact that we can write $\mathbf{W}_{0}^{H}\left(\mathbf{I}_{M}-\mathbf{S}_{1}\right) \mathbf{W}_{0}$ as $\mathbf{W}_{0}^{H}\left(\mathbf{I}_{M}-\mathbf{S}_{1}\right)^{H}\left(\mathbf{I}_{M}-\mathbf{S}_{1}\right) \mathbf{W}_{0}$, we can 
conclude that the columns of $\left(\mathbf{I}_{M}-\mathbf{S}_{1}\right) \mathbf{W}_{0}$ must be orthogonal to each other. We at this point assume that the total number of subcarriers $M$ is a power of two. Then, if we choose $K$ to be also a power of two, satisfying $K \geq L_{c}-L-1$, and place the unused subcarriers $M / K$ subcarriers apart, the nonzero entries of $\left(\mathbf{I}_{M}-\mathbf{S}_{1}\right) \mathbf{W}_{0}$ form the first $L_{c}-L-1$ (rotated) column vectors of a size $K$ DFT matrix and are thus orthogonal. The entries of the carrier selection matrix $\boldsymbol{S}_{1}$ are thus given by the following solution, where $j$ is an integer value with $0 \leq j<M / K$ :

$$
s_{i}= \begin{cases}0, & \text { if } i=j+\frac{\ell M}{K}, \quad \ell=0,1, \ldots, K-1 . \\ 1, & \text { otherwise, }\end{cases}
$$

Taking further into consideration that in a DMT setting, the data in subcarrier $M-k$ is the complex conjugate of the data in subcarrier $k$, with $k=1, \ldots, M / 2-1$, in order to guarantee real-valued data at the output of the transmitter, only two choices for $j$ in (35) remain to place unused subcarriers. These are $j=0$ and $j=M / 2 K$. For these solutions, we obtain

$$
\mathbf{W}_{0}^{H}\left(\mathbf{I}_{M}-\mathbf{S}_{1}\right) \mathbf{W}_{0}=K \cdot \mathbf{I}_{L_{c}-L-1} .
$$

Substituting this result into (31), the noise variance and SNR in a used subcarrier $k$ yields

$$
\begin{aligned}
& \sigma_{n, k}^{2}=\frac{\sigma_{r}^{2}}{\left|C\left(e^{j(2 \pi k / M)}\right)\right|^{2}}\left(1+\frac{L_{c}-L-1}{K}\right), \\
& \mathrm{SNR}_{k}=\frac{\sigma_{u, k}^{2}}{\sigma_{n, k}^{2}}=\frac{\sigma_{u, k}^{2} \cdot\left|C\left(e^{j(2 \pi k / M)}\right)\right|^{2}}{\sigma_{r}^{2}\left(1+\left(L_{c}-L-1\right) / K\right)} .
\end{aligned}
$$

Here, the SNRs in used subcarriers depend again not only on the channel magnitude frequency response and the number of unused subcarriers but also on the number of samples by which the guard interval is too short. Since the number of combinations for the placement of the unused subcarriers has been significantly reduced, an extensive search can be performed to find the placement resulting in the highest data rate. Starting with the smallest power of two greater than $L_{c}-L-1$ for $K$, the optimal value for $j$ in (35) can be determined. Then, $K$ is doubled and the search for an optimal $j$ is performed again. This procedure is repeated while the data rate keeps increasing.

\subsection{General case}

The noise variance at the equalizer output for a general placement of $K$ unused subcarriers is given in (31). Since it depends on the carrier selection matrix $S_{1}$, the noise variance can only be calculated once a decision has been made on which subcarriers should remain unused, not knowing how good this decision is. Some better insight can be gained when reformulating (31) using the matrix inversion lemma [33], see Appendix B for details.

$$
\begin{aligned}
\operatorname{diag} & \left(\left[\sigma_{n, 0}^{2}, \sigma_{n, 1}^{2}, \ldots, \sigma_{n, M-1}^{2}\right]\right) \\
= & \sigma_{r}^{2} \mathbf{C}_{\text {freq }}^{\dagger}\left(\mathbf{C}_{\text {freq }}^{\dagger}\right)^{H} \mathbf{S}_{1} \\
& \cdot\left(\mathbf{I}_{M}+\operatorname{diag}\left(\frac{\mathbf{W}_{0} \mathbf{W}_{0}^{H}}{M}\left(\mathbf{I}_{M}-\mathbf{S}_{1} \frac{\mathbf{W}_{0} \mathbf{W}_{0}^{H}}{M}\right)^{-1}\right)\right) \\
= & \sigma_{r}^{2} \mathbf{C}_{\text {freq }}^{\dagger}\left(\mathbf{C}_{\text {freq }}^{\dagger}\right)^{H} \\
& \cdot\left(\mathbf{S}_{1}-\mathbf{I}_{M}+\operatorname{diag}\left(\left(\mathbf{I}_{M}-\mathbf{S}_{1} \frac{\mathbf{W}_{0} \mathbf{W}_{0}^{H}}{M}\right)^{-1}\right)\right) .
\end{aligned}
$$

The noise variance and SNR in a used subcarrier $k$ thus yield

$$
\begin{gathered}
\sigma_{n, k}^{2}=\frac{\sigma_{r}^{2}}{\left|C\left(e^{j(2 \pi k / M)}\right)\right|^{2}}\left[\operatorname{diag}\left(\left(\mathbf{I}_{M}-\mathbf{S}_{1} \frac{\mathbf{W}_{0} \mathbf{W}_{0}^{H}}{M}\right)^{-1}\right)\right]_{k, k}, \\
\mathrm{SNR}_{k}=\frac{\sigma_{u, k}^{2}}{\sigma_{n, k}^{2}}=\frac{\sigma_{u, k}^{2} \cdot\left|C\left(e^{j(2 \pi k / M)}\right)\right|^{2}}{\sigma_{r}^{2}\left[\operatorname{diag}\left(\left(\mathbf{I}_{M}-\mathbf{S}_{1}\left(\mathbf{W}_{0} \mathbf{W}_{0}^{H} / M\right)\right)^{-1}\right)\right]_{k, k}} .
\end{gathered}
$$

For $\left\|\mathbf{S}_{1} \mathbf{W}_{0} \mathbf{W}_{0}^{H} / M\right\|<1$, the inverse matrix can be expressed using the Neumann expansion [33],

$$
\left(\mathbf{I}_{M}-\mathbf{S}_{1} \frac{\mathbf{W}_{0} \mathbf{W}_{0}^{H}}{M}\right)^{-1}=\sum_{i=0}^{\infty}\left(\mathbf{S}_{1} \frac{\mathbf{W}_{0} \mathbf{W}_{0}^{H}}{M}\right)^{i},
$$

and thus approximated through a finite series. A possible approach is to use only a few terms of the series to determine $S_{1}$. We can afterwards verify the quality of the approximation by substituting the matrix $\boldsymbol{S}_{1}$ derived in this way into (39) comparing this result with the one obtained from the approximation.

\section{SIMULATION RESULTS}

The performance of the proposed frequency domain ZF equalizer was evaluated through simulation of a DMT transceiver in Matlab. The block length is $M=128$ and the target bit error rate is set to $10^{-6}$. The discrete channel impulse response, sampled at $f_{s}=1.024 \mathrm{MHz}$, was obtained through actual measurement of a twisted-pair copper wire of $4 \mathrm{~km}$ length and a diameter of $0.8 \mathrm{~mm}$. For simulation purposes, the impulse response has been artificially shortened to 35 taps, removing a tail of very small values. The impulse response and the magnitude frequency response are shown in Figure 7.

AWGN channel noise $r(n)$ with different variances $\sigma_{r}^{2}$ was applied. The transmit signal power $\sigma_{u}^{2}$ was set to be a $1 / M$, that is, $\sum_{i=0}^{M-1} \sigma_{u_{i}}^{2}=1$. Thus the more subcarriers are used, the less power is available per subcarrier. The bitload and power per subcarrier is calculated in adaptive loading algorithm [26] using the SNRs for the ZF equalizer as described 


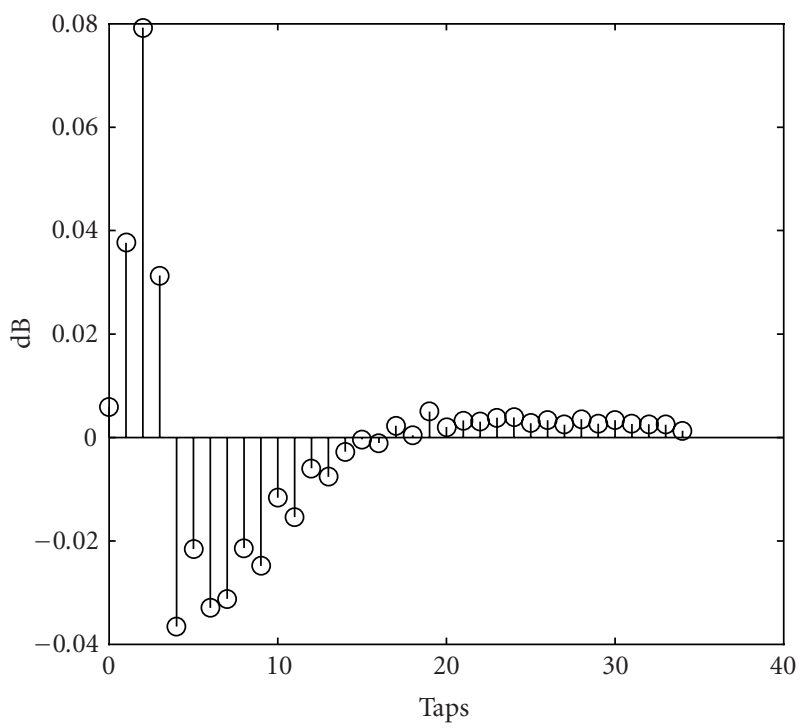

(a)

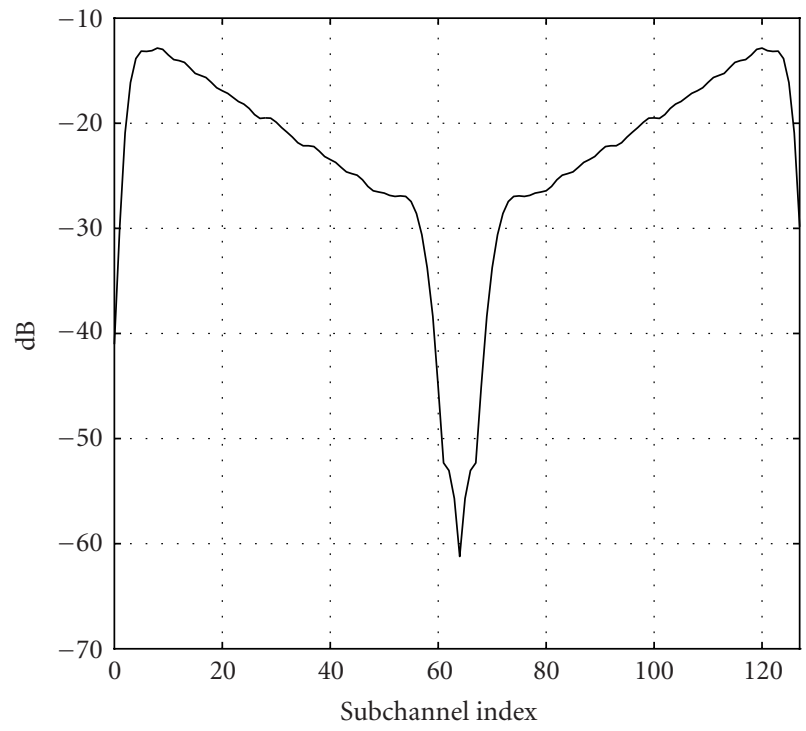

(b)

FIGURE 7: (a) Channel impulse response and (b) magnitude frequency response.

in the previous section for the different cases. Denoting the sampling rate at the transmitter output $f_{s}=1 / T$, the bitrate is calculated as

$$
\text { bitrate }=\frac{f_{s}}{M+L} \sum_{k=1}^{M / 2-1} b_{k}
$$

where $b_{k}$ are the bits per subcarrier determined by the adaptive loading algorithm. Only an even number of bits is assigned per subcarrier in order to stay with square QAM constellations.

\subsection{Example 1}

In a first simulation, we investigate the case where the guard interval is just one tap too short ( $L=33$ taps, $K \geq 1$ ). For several noise variances, the number of unused subcarriers $K$ has been varied. The $K$ subcarriers with the lowest SNR according to (34) are assigned as unused subcarriers. The normalized bitrates are shown in Figure 8. The value given for $K=0$ is the data rate achievable with a guard interval of sufficient length and is shown for comparison.

The maximum data rate occurs for values of $K$ greater than the required $K=1$, since the noise variance at the receiver output reduces with increasing $K$. The higher the noise variance, the larger is the optimum value for $K$ since an increasing number of subcarriers remains unloaded even in DMT with sufficient guard interval due to a too low SNR.

\subsection{Example 2}

In a second set of simulations, we space the unused subcarriers equidistantly (see Section 5.2). Thus, the number of unused subcarriers $K$ is restricted to be a power of two. The

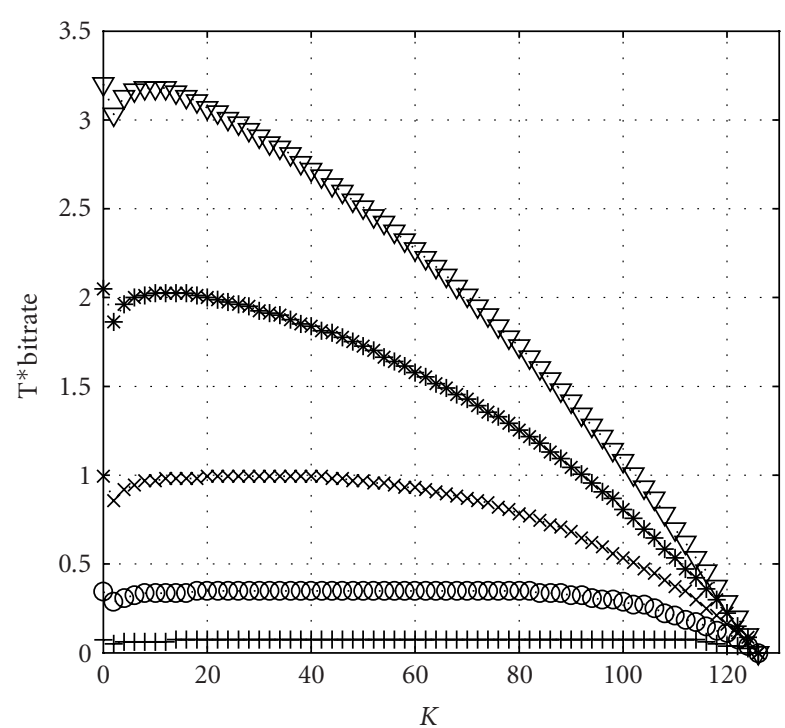

Figure 8: Bitrates for $10 \log _{10}\left(\sigma_{u}^{2} / \sigma_{r}^{2}\right)=20 \mathrm{~dB}(+), 30 \mathrm{~dB}(\circ)$, $40 \mathrm{~dB}(\times), 50 \mathrm{~dB}(*)$, and $60 \mathrm{~dB}(\nabla)$ if the guard interval is one tap too short.

guard interval is varied from $L=L_{c}-2$ to $L=0$. Figure 9 shows the achievable data rate for all possible values of $K$ dependent on the number of taps by which the guard interval is too short.

For $10 \log _{10}\left(\sigma_{u}^{2} / \sigma_{r}^{2}\right)=30 \mathrm{~dB}$, only a few subcarriers are loaded even in the case of a guard interval of sufficient length. Thus, by increasing $K$ we do not sacrify many good subcarriers and the overall data rate increases since the noise enhancement is inverse proportional to $K$, see (38). For 


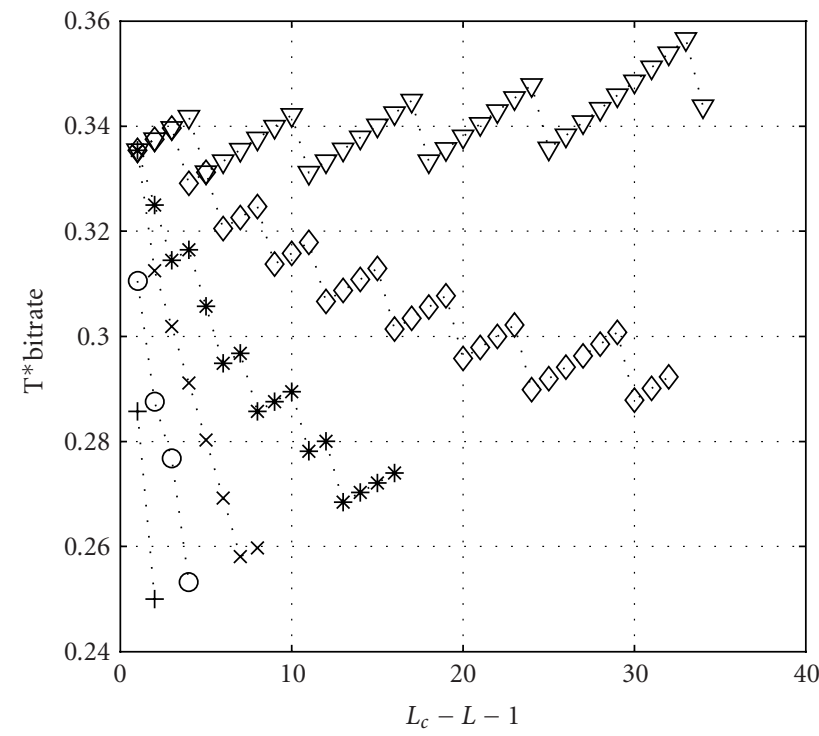

(a)

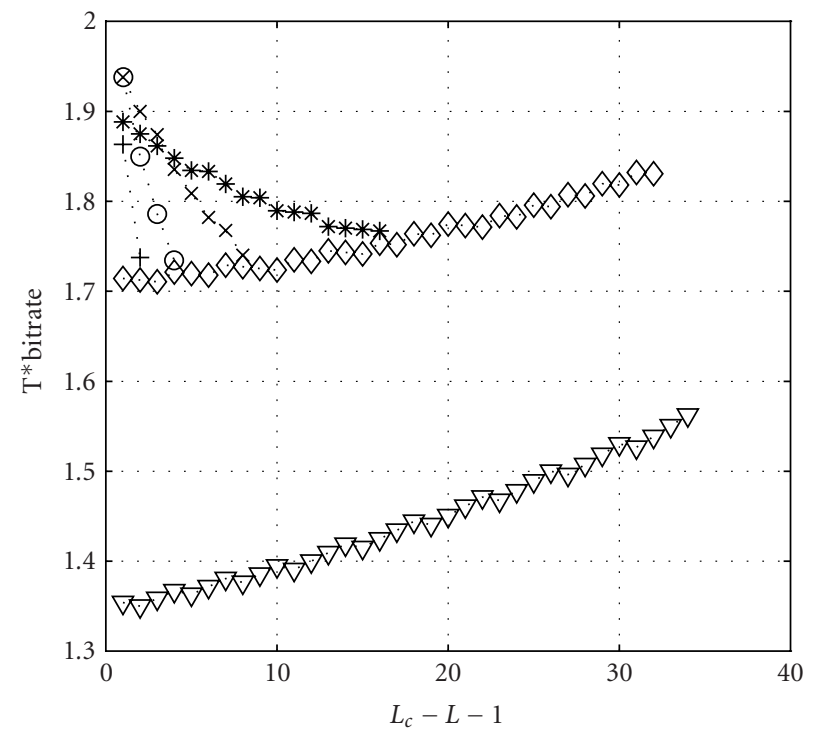

(b)

Figure 9: (a) Normalized bitrates for $10 \log _{10}\left(\sigma_{u}^{2} / \sigma_{r}^{2}\right)=30 \mathrm{~dB}$ and (b) $50 \mathrm{~dB} . K=2(+), 4(\circ), 8(\times), 16(*), 32(\diamond), 64(\nabla)$.

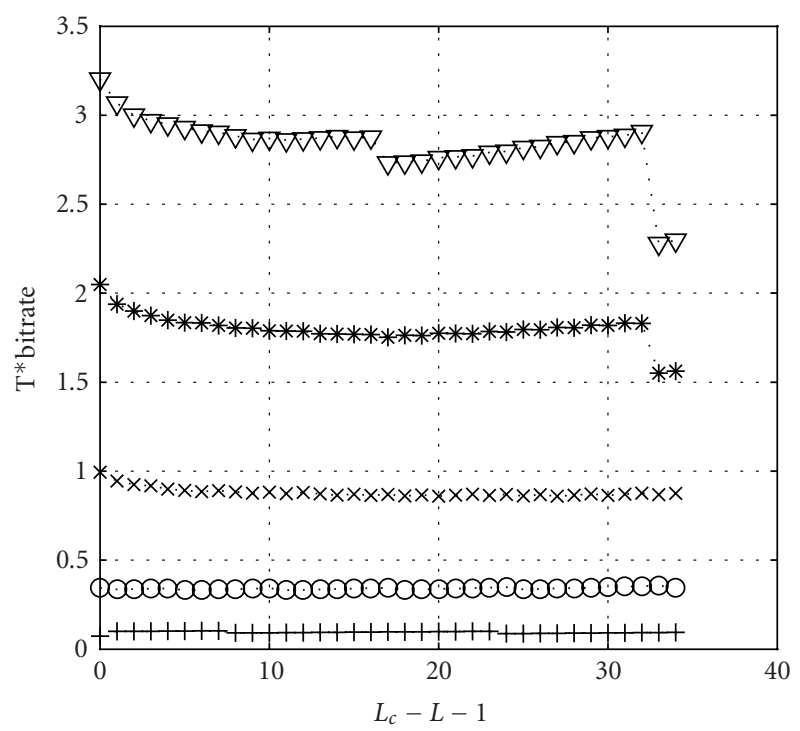

Figure 10: Highest bitrates for $10 \log _{10}\left(\sigma_{u}^{2} / \sigma_{r}^{2}\right)=20 \mathrm{~dB}(+)$, $30 \mathrm{~dB}(\circ), 40 \mathrm{~dB}(\times), 50 \mathrm{~dB}(*), 60 \mathrm{~dB}(\nabla)$ assigning unused subcarriers equidistantly.

$10 \log _{10}\left(\sigma_{u}^{2} / \sigma_{r}^{2}\right)=50 \mathrm{~dB}$, we observe a reduction of the data rate when using $K=32$ and $K=64$, since too many subcarriers that carry bits in traditional DMT have to be reserved as unused subcarriers. Taking the maximum bitrate of all $K$ for each guard interval length results in Figure 10. The data rate for $L_{c}-L-1=0$ is the one resulting from traditional DMT with a guard interval of sufficient length.
We observe that the data rate reduces only slightly for moderate SNRs. Reducing the guard interval results in a lower latency time of the system. In applications where latency time is a predominant concern, the reduction in bitrate might be acceptable.

\subsection{Example 3}

In this example, we assign the $K$ subcarriers with the highest channel frequency attenuation to be the unused subcarriers. These are the subcarriers that a traditional DMT transceiver would leave unused in case the allowed total transmit power is not high enough to assign bits to them. As we can see from the channel frequency response in Figure 7, the unused subcarriers form two blocks: one at low frequencies and one at high frequencies. $K$ is varied for each value of $L$ from $L_{c}-L-1$ to $M$ in steps of one, hoping that the extra redundancy introduced reduces the noise enhancement. Then the $K$ with the highest data rate is chosen, resulting in Figure 11.

For small values of $L_{c}-L-1$, we obtain higher data rates than in the case of equidistant spacing of unused subcarriers. However, as $L_{c}-L-1$ increases, the data rate reduces dramatically for $10 \log _{10}\left(\sigma_{u}^{2} / \sigma_{r}^{2}\right)>30 \mathrm{~dB}$. This is because the denominator in (42) depends on the position of the unused subcarriers and grows fast for the choice made here.

\subsection{Example 4}

In a last example, we have shortened the channel impulse response using a time domain equalizer with 20 taps. The TEQ coefficients were designed such that the mean squared error of the target impulse response outside the desired window is minimized, see [2]. The delay of the window was optimally chosen for all desired channel impulse response 


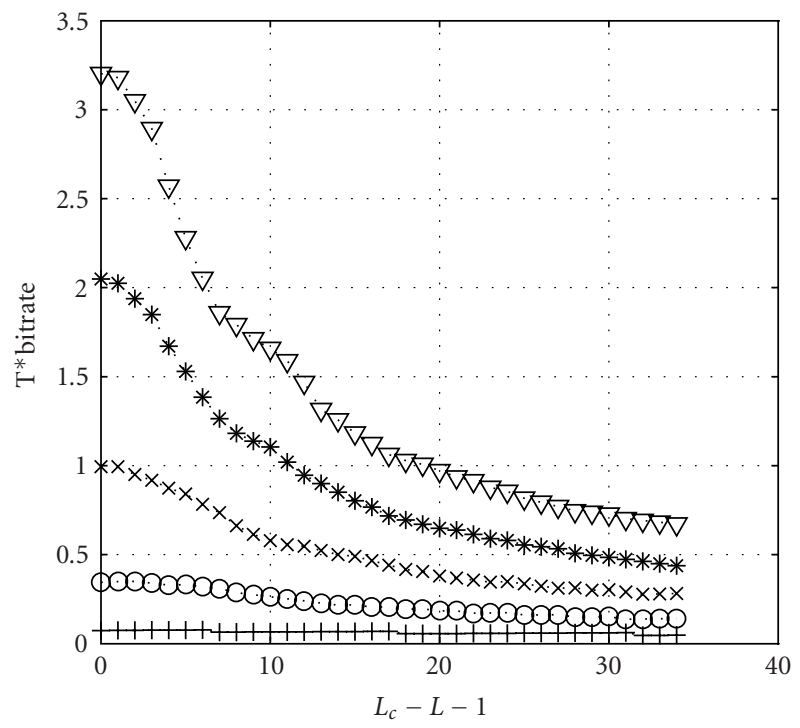

FIgURE 11: Highest bitrates for $10 \log _{10}\left(\sigma_{u}^{2} / \sigma_{r}^{2}\right)=20 \mathrm{~dB}(+)$, $30 \mathrm{~dB}(\circ), 40 \mathrm{~dB}(\times), 50 \mathrm{~dB}(*), 60 \mathrm{~dB}(\nabla)$ choosing the subcarriers with the highest channel frequency attenuation to be the unused subcarriers.

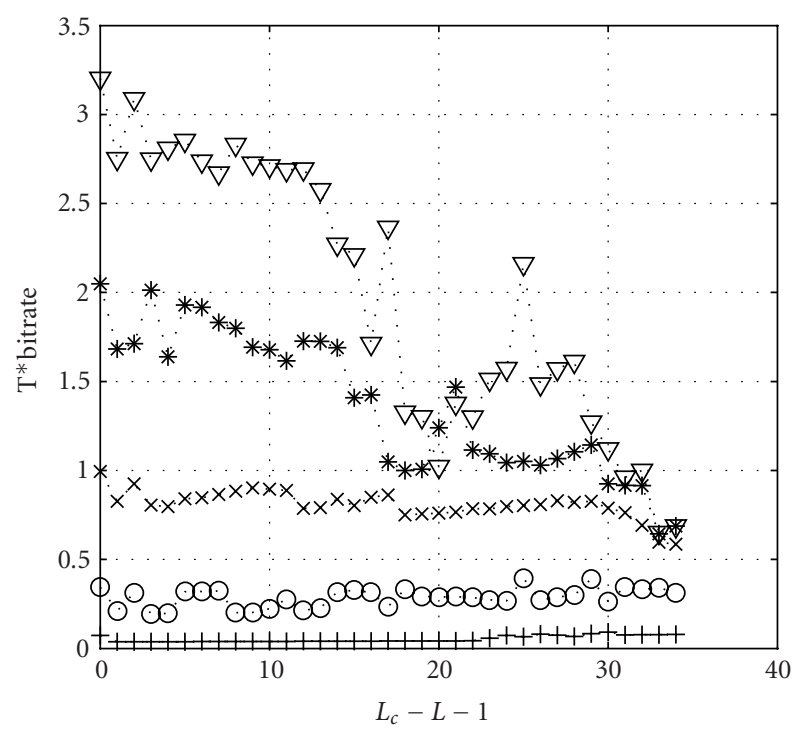

Figure 12: Highest bitrates for $10 \log _{10}\left(\sigma_{u}^{2} / \sigma_{r}^{2}\right)=20 \mathrm{~dB}(+)$, $30 \mathrm{~dB}(\circ), 40 \mathrm{~dB}(\times), 50 \mathrm{~dB}(*), 60 \mathrm{~dB}(\nabla)$ with TEQ channel impulse response shortening.

lengths. Since the impulse response resulting from convolution of the channel with the TEQ filter has nonzero taps outside the window, the SNRs also take into account the resulting interference.

Comparing Figure 12 with Figures 10 and 11, we observe that the TEQ performs better than our suboptimal approach, but results in lower data rates than the equidistant spacing of unused subcarriers, particularly for short guard intervals.

\section{CONCLUSION}

In this paper, we have presented a new ZF frequency domain block equalizer for DMT transceivers with insufficient guard interval. The equalizer takes advantage of unused subcarriers and allows to trade off time domain redundancy for frequency domain redundancy, thus resulting at a lower transceiver latency time for the same data rate. We have given sufficient conditions for ZF equalization and shown that the only nonzero entries in the block equalizer are the ones that are already present in conventional DMT plus additional branches that combine used subcarriers with unused subcarriers. The equalizer thus utilizes the ISI/ICI leakage caused by the FFT operation at the receiver side. The noise enhancement of the equalizer has been calculated. For each subcarrier, it depends on the channel attenuation in the subcarrier, as well as the placement of all unused subcarriers. The noise variance at the receiver output has been calculated for guard intervals that are just one tap too short and for equidistant spacing of unused subcarriers. For the general case, an expression for the noise variance has been derived that still depends on the placement of the unused subcarriers. Finding an optimal solution to this problem is the focus of future studies. As simulations have shown, it is not as easy as to assign the subcarriers with the highest channel attenuation as unused subcarriers. The redundance needed for ZF equalization is the same as in traditional DMT, however, it is placed here in such a way that the system latency time can be reduced. Also, simulations have shown that the solution with the lowest redundancy does not necessarily result in the highest data rate. This might change when calculating the equalizer coefficients according to an MSE criterion instead of ZF and is also the scope of future studies.

\section{APPENDICES}

\section{A. NOISE VARIANCE}

In order to derive the noise variance at the output of each subband, we first calculate $\mathbf{E}_{0} \mathbf{E}_{0}^{H}$ applying (26).

$$
\begin{aligned}
\mathbf{E}_{0} \mathbf{E}_{0}^{H}= & \left(\mathbf{S}_{1} \mathbf{C}_{\text {freq }}^{\dagger} \mathbf{W}_{0}\left(\left(\mathbf{I}_{M}-\mathbf{S}_{1}\right) \mathbf{W}_{0}\right)^{\dagger}\right) \\
& \cdot\left(\mathbf{S}_{1} \mathbf{C}_{\text {freq }}^{\dagger} \mathbf{W}_{0}\left(\left(\mathbf{I}_{M}-\mathbf{S}_{1}\right) \mathbf{W}_{0}\right)^{\dagger}\right)^{H} .
\end{aligned}
$$

For reasons of conciseness, we introduce the abbreviation

$$
\mathbf{A}=\left(\left(\mathbf{I}_{M}-\mathbf{S}_{1}\right) \mathbf{W}_{0}\right),
$$

yielding

$$
\mathbf{E}_{0} \mathbf{E}_{0}^{H}=\mathbf{S}_{1} \mathbf{C}_{\text {freq }}^{\dagger} \mathbf{W}_{0} \mathbf{A}^{\dagger} \mathbf{A}^{\dagger}{ }^{H} \mathbf{W}_{0}^{H} \mathbf{C}_{\text {freq }}^{\dagger}{ }^{H} \mathbf{S}_{1} .
$$

Using singular value decomposition, one can easily show that $\mathbf{A}^{\dagger}\left(\mathbf{A}^{\dagger}\right)^{H}=\left(\mathbf{A}^{H} \mathbf{A}\right)^{\dagger}$ and with $\mathbf{A}$ from (A.2), this yields

$$
\begin{aligned}
\left(\mathbf{A}^{H} \mathbf{A}\right)^{\dagger} & =\left(\left(\left(\mathbf{I}_{M}-\mathbf{S}_{1}\right) \mathbf{W}_{0}\right)^{H}\left(\left(\mathbf{I}_{M}-\mathbf{S}_{1}\right) \mathbf{W}_{0}\right)\right)^{\dagger} \\
& =\left(\mathbf{W}_{0}^{H}\left(\mathbf{I}_{M}-\mathbf{S}_{1}\right) \mathbf{W}_{0}\right)^{\dagger}=\left(\mathbf{W}_{0}^{H}\left(\mathbf{I}_{M}-\mathbf{S}_{1}\right) \mathbf{W}_{0}\right)^{-1}
\end{aligned}
$$


and since the resulting matrix is square and always of full rank, taking the pseudoinverse was replaced by taking the inverse in the last step. We now have

$$
\mathbf{E}_{0} \mathbf{E}_{0}^{H}=\mathbf{S}_{1} \mathbf{C}_{\text {freq }}^{\dagger} \mathbf{W}_{0}\left(\mathbf{W}_{0}^{H}\left(\mathbf{I}_{M}-\mathbf{S}_{1}\right) \mathbf{W}_{0}\right)^{-1} \mathbf{W}_{0}^{H} \mathbf{C}_{\text {freq }}^{\dagger}{ }^{H} \mathbf{S}_{1} .
$$

Once we have taken the diagonal of this expression (taking into consideration that $\mathbf{S}_{1}$ and $\mathbf{C}_{\text {freq }}^{\dagger}$ are already diagonal matrices), each factor is a diagonal matrix and the order of the matrices can be interchanged. With $\mathbf{S}_{1} \cdot \mathbf{S}_{1}=\mathbf{S}_{1}$ we obtain

$$
\begin{aligned}
& \operatorname{diag}\left(\mathbf{E}_{0} \mathbf{E}_{0}^{H}\right) \\
& \quad=\mathbf{C}_{\text {freq }}^{\dagger}\left(\mathbf{C}_{\text {freq }}^{\dagger}\right)^{H} \mathbf{S}_{1} \operatorname{diag}\left(\mathbf{W}_{0}\left(\mathbf{W}_{0}^{H}\left(\mathbf{I}_{M}-\mathbf{S}_{1}\right) \mathbf{W}_{0}\right)^{-1} \mathbf{W}_{0}^{H}\right),
\end{aligned}
$$

$\operatorname{diag}\left(\mathbf{E}_{1} \mathbf{E}_{1}^{H}\right)$ can be easily obtained from (21) as

$$
\operatorname{diag}\left(\mathbf{E}_{1} \mathbf{E}_{1}^{H}\right)=\mathbf{C}_{\text {freq }}^{\dagger}\left(\mathbf{C}_{\text {freq }}^{\dagger}\right)^{H} \mathbf{S}_{1}
$$

Adding (A.6) and (A.7) and multiplying it with $\sigma_{r}^{2}$ results in (31).

\section{B. MATRIX INVERSION LEMMA}

The matrix inversion lemma [33] states that

$$
(\mathbf{A}+\mathbf{X R Y})^{-1}=\mathbf{A}^{-1}-\mathbf{A}^{-1} \mathbf{X}\left(\mathbf{R}^{-1}+\mathbf{Y A}^{-1} \mathbf{X}\right)^{-1} \mathbf{Y A}^{-1},
$$

where $\mathbf{A}$ is a regular $n \times n$ matrix, $\mathbf{X}$ is $n \times r, \mathbf{Y}$ is $r \times n$, and $\mathbf{R}$ is a regular $r \times r$ matrix. A useful identity that can be derived from (B.1) is

$$
(\mathbf{A}+\mathbf{X R Y})^{-1} \mathbf{X R}=\mathbf{A}^{-1} \mathbf{X}\left(\mathbf{R}^{-1}+\mathbf{Y A}^{-1} \mathbf{X}\right)^{-1} .
$$

Choosing $\mathbf{A}=M \cdot \mathbf{I}_{L-L_{c}-1}, \mathbf{X}=\mathbf{W}_{0}^{H}, \mathbf{R}=\mathbf{I}_{M}, \mathbf{Y}=-\mathbf{S}_{1} \mathbf{W}_{0}$ yields

$$
\begin{aligned}
\mathbf{W}_{0} & \left(\mathbf{W}_{0}^{H}\left(\mathbf{I}_{M}-\mathbf{S}_{1}\right) \mathbf{W}_{0}\right)^{-1} \mathbf{W}_{0}^{H} \\
= & \mathbf{W}_{0}\left(\mathbf{W}_{0}^{H} \mathbf{W}_{0}-\mathbf{W}_{0}^{H} \mathbf{S}_{1} \mathbf{W}_{0}\right)^{-1} \mathbf{W}_{0}^{H} \\
= & \mathbf{W}_{0}\left(M \cdot \mathbf{I}_{L-L_{c}-1}-\mathbf{W}_{0}^{H} \mathbf{S}_{1} \mathbf{W}_{0}\right)^{-1} \mathbf{W}_{0}^{H} \\
= & \mathbf{W}_{0} \frac{\mathbf{I}_{L-L_{c}-1}}{M} \mathbf{W}_{0}^{H}\left(\mathbf{I}_{M}-\mathbf{S}_{1} \mathbf{W}_{0} \frac{\mathbf{I}_{L-L_{c}-1}}{M} \mathbf{W}_{0}^{H}\right)^{-1} \\
= & \frac{\mathbf{W}_{0} \mathbf{W}_{0}^{H}}{M}\left(\mathbf{I}_{M}-\mathbf{S}_{1} \frac{\mathbf{W}_{0} \mathbf{W}_{0}^{H}}{M}\right)^{-1},
\end{aligned}
$$

where (B.2) has been applied to obtain (B.6) from (B.5). Applying these modifications to (31) directly results in (39). We can further simplify (39) through the following modifications that result in (40):

$$
\begin{gathered}
\mathbf{S}_{1}\left(\mathbf{I}_{M}+\operatorname{diag}\left(\frac{\mathbf{W}_{0} \mathbf{W}_{0}^{H}}{M}\left(\mathbf{I}_{M}-\mathbf{S}_{1} \frac{\mathbf{W}_{0} \mathbf{W}_{0}^{H}}{M}\right)^{-1}\right)\right) \\
=\mathbf{S}_{1}+\operatorname{diag}\left(\mathbf{S}_{1} \frac{\mathbf{W}_{0} \mathbf{W}_{0}^{H}}{M}\left(\mathbf{I}_{M}-\mathbf{S}_{1} \frac{\mathbf{W}_{0} \mathbf{W}_{0}^{H}}{M}\right)^{-1}\right) \\
=\mathbf{S}_{1}+\operatorname{diag}\left(\left(\mathbf{I}_{M}-\mathbf{S}_{1} \frac{\mathbf{W}_{0} \mathbf{W}_{0}^{H}}{M}\right)^{-1}-\mathbf{I}_{M}\right) \\
=\mathbf{S}_{1}-\mathbf{I}_{M}+\operatorname{diag}\left(\left(\mathbf{I}_{M}-\mathbf{S}_{1} \frac{\mathbf{W}_{0} \mathbf{W}_{0}^{H}}{M}\right)^{-1}\right)
\end{gathered}
$$

To obtain (B.10) from (B.9), we have used the fact that

$$
\begin{aligned}
\mathbf{B}(\mathbf{I}-\mathbf{B})^{-1} & =((\mathbf{B}-\mathbf{I})+\mathbf{I})(\mathbf{I}-\mathbf{B})^{-1} \\
& =-(\mathbf{I}-\mathbf{B})(\mathbf{I}-\mathbf{B})^{-1}+\mathbf{I}(\mathbf{I}-\mathbf{B})^{-1} \\
& =-\mathbf{I}+(\mathbf{I}-\mathbf{B})^{-1} .
\end{aligned}
$$

\section{REFERENCES}

[1] W. Goralski, ADSL and DSL Technologies, McGraw-Hill, New York, NY, USA, 1998.

[2] N. Al-Dhahir and J. M. Cioffi, "Optimum finite-length equalization for multicarrier transceivers," IEEE Trans. Communications, vol. 44, no. 1, pp. 56-64, 1996.

[3] P. J. W. Melsa, R. C. Younce, and C. E. Rohrs, "Impulse response shortening for discrete multitone transceivers," IEEE Trans. Communications, vol. 44, no. 12, pp. 1662-1672, 1996.

[4] M. Nafie and A. Gatherer, "Time-domain equalizer training for ADSL," in Proc. IEEE International Conference on Communications, vol. 2, pp. 1085-1089, Montreal, Quebec, Canada, June 1997.

[5] N. Lashkarian and S. Kiaei, "Fast algorithm for finite-length MMSE equalizers with application to discrete multitone systems," in Proc. IEEE International Conference on Acoustics, Speech, and Signal Processing, vol. 5, pp. 2753-2756, Phoenix, Ariz, USA, March 1999.

[6] G. Arslan, B. L. Evans, and S. Kiaei, "Equalization for discrete multitone transceivers to maximize bit rate," IEEE Trans. Signal Processing, vol. 49, no. 12, pp. 3123-3135, 2001.

[7] K. Van Acker, G. Leus, M. Moonen, O. van de Wiel, and T. Pollet, "Per tone equalization for DMT-based systems," IEEE Trans. Communications, vol. 49, no. 1, pp. 109-119, 2001.

[8] X.-G. Xia, "New precoding for intersymbol interference cancellation using nonmaximally decimated multirate filterbanks with ideal FIR equalizers," IEEE Trans. Signal Processing, vol. 45, no. 10, pp. 2431-2441, 1997.

[9] L. Vandendorpe, L. Cuvelier, F. Deryck, J. Louveaux, and O. van de Wiel, "Fractionally spaced linear and decisionfeedback detectors for transmultiplexers," IEEE Trans. Signal Processing, vol. 46, no. 4, pp. 996-1011, 1998.

[10] X. Lin and A. N. Akansu, "Nonmaximally decimated filterbank based precoder/post-equalizer for blind channel identification and optimal MMSE equalization," in Proc. IEEE 
International Conference on Acoustics, Speech, and Signal Processing, vol. 6, pp. 3505-3508, Seattle, Wash, USA, May 1998.

[11] T. Li and Z. Ding, "Joint transmitter-receiver optimization for partial response channels based on nonmaximally decimated filterbank precoding technique," IEEE Trans. Signal Processing, vol. 47, no. 9, pp. 2407-2414, 1999.

[12] A. Scaglione, G. B. Giannakis, and S. Barbarossa, "Redundant filterbank precoders and equalizers. I. Unification and optimal designs," IEEE Trans. Signal Processing, vol. 47, no. 7, pp. 1988-2006, 1999.

[13] S. Trautmann, T. Karp, and N. J. Fliege, "Comparing TEQ and MMSE receivers for short-latency DMT transmission," in Proc. 1st International OFDM-Workshop, Hamburg, Germany, September 1999.

[14] R. E. Crochiere and L. R. Rabiner, Multirate Digital Signal Processing, Prentice-Hall, Englewood Cliffs, NJ, USA, 1983.

[15] P. P. Vaidyanathan, Multirate Systems and Filter Banks, Prentice Hall, Englewood Cliffs, NJ, USA, 1993.

[16] N. Suzuki, H. Uehara, and M. Yokoyama, "A new OFDM demodulation method to reduce influence of ISI due to longer delay than guard interval," in Proc. 8th IEEE International Conference on Communication Systems, vol. 1, pp. 239-244, Singapore, November 2002.

[17] S. Trautmann and N. J. Fliege, "A new equalizer for multitone systems without guard time," IEEE Communications Letters, vol. 6, no. 1, pp. 34-36, 2002.

[18] S. Trautmann and N. J. Fliege, "Perfect equalization for DMT systems without guard interval," IEEE Journal on Selected Areas in Communications, vol. 20, no. 5, pp. 987-996, 2002.

[19] A. Gatherer and M. Polley, "Controlling clipping probability in DMT transmission," in Proc. 31st Asilomar Conference on Signals, Systems and Computers, vol. 1, pp. 578-584, Pacific Grove, Calif, USA, November 1997.

[20] J. Tellado and J. M. Cioffi, "Efficient algorithms for reducing PAR in multicarrier systems," in Proc. IEEE International Symposium on Information Theory, p. 191, Cambridge, Mass, USA, August 1998.

[21] J. Tellado and J. M. Cioffi, "PAR reduction in multicarrier transmission systems," ANSI T1E1.4 contribution number 97-367, Sacramento, Calif, USA, December 1997 and ITU Study Group 15, contribution number D150 (WP 1/15), Geneva, Switzerland, February 1998.

[22] J. Tellado and J. M. Cioffi, "Revisiting DMT's PAR," ETSI/ANSI TM6 contribution number TD08, Antwerp, Belgium, April 1998 and ANSI T1E1.4 contribution number 98083, Austin, Tex, USA, March 1998.

[23] J. Tellado and J. M. Cioffi, "Further results on peak-toaverage ratio reduction," ANSI T1E1.4 contribution number 98-252, San Antonio, Tex, USA, August 1998 and ETSI/ANSI TM6 contribution number TD15, Vienna, Austria, September 1998.

[24] T. Karp, S. Trautmann, and N. J. Fliege, "Frequency domain equalization of DMT/OFDM systems with insufficient guard interval," in Proc. IEEE International Conference on Communications, vol. 3, pp. 1646-1650, New York, NY, USA, April 2002.

[25] J. M. Cioffi, A multicarrier primer, http://www-isl.stanford. edu/ cioffi/papers.html.

[26] D. Hughes-Hartog, "Ensemble modem structure for imperfect transmission media," US patent 4,679,227 (July 1987), US patent 4,731,816 (March 1988), and US patent 4,833,706 (May 1989).

[27] J. Campello, "Optimal discrete bit loading for multicarrier modulation systems," in Proc. IEEE International Symposium on Information Theory, p. 193, Cambridge, Mass, USA, August 1998.

[28] J. Campello, "Practical bit loading for DMT" in Proc. IEEE International Conference on Communications, vol. 2, pp. 801805, Vancouver, BC, Canada, June 1999.

[29] B. Farhang-Boroujeny and M. Ding, "An eigen-approach to the design of near-optimum time domain equalizer for DMT transceivers," in Proc. IEEE International Conference on Communications, vol. 2, pp. 937-941, Vancouver, BC, Canada, June 1999.

[30] B. Wang and T. Adali, "Joint impulse response shortening for discrete multitone systems," in Proc. IEEE Global Telecommunications Conference, vol. 5, pp. 2508-2512, Rio de Janeiro, Brazil, December 1999.

[31] B. Wang and T. Adali, "Time-domain equalizer design for discrete multitone systems," in Proc. IEEE International Conference on Communications, vol. 2, pp. 1080-1084, New Orleans, La, USA, June 2000.

[32] R. V. Sonalkar and R. R. Shively, "An efficient bit-loading algorithm for DMT applications," IEEE Communications Letters, vol. 4, no. 3, pp. 80-82, 2000.

[33] T. K. Moon and W. C. Stirling, Mathematical Methods and Algorithms for Signal Processing, Prentice-Hall, Upper Saddle River, NJ, USA, 2000.

Tanja Karp received the Dipl.-Ing. degree in electrical engineering (M.S.E.E.) and the Dr.-Ing. degree (Ph.D.) from Technical University Hamburg-Harburg, Hamburg, Germany, in 1993 and 1997, respectively. In 1995 and 1996, she spent two months as a Visiting Researcher at the Signal Processing Department of ENST, Paris, France, and at the Mutirate Signal Processing Group, University of Wisconsin, Madison, respectively, working on modulated filter banks. In 1997, she joined the Institute of Computer Engineering at Mannheim University, Germany, as a Senior Research and Teaching Associate. From 1998 to 1999, she had also taught as a Guest Lecturer at the Institute for Microsystem Technology at Freiburg University, Germany. Since 2000, she has been an Assistant Professor in the Department of Electrical and Computer Engineering at Texas Tech University, Lubbock, Texas. Her research interests include multirate signal processing, filter banks, audio coding, multicarrier modulation, multiple description coding, and signal processing for communications. Dr. Karp is an IEEE member and regularly reviews articles for several IEEE and EURASIP transactions.

Steffen Trautmann received the Dipl.-Ing. degree in information technology from the Dresden University of Technology, Dresden, Germany, in 1996. From October 1994 to April 1995, he was a Visiting Student at the University of Wisconsin, Madison, USA. He was a Ph.D. Student at the Hamburg University of Technology, Hamburg, Germany, from February 1996 to December 1996. From 1997 to 2001, he was with the Mannheim University, Mannheim, Germany, as a Research and Teaching Assistant. After finishing his Ph.D. thesis, he was a Senior Researcher at the Telecommunications Research Center Vienna (ftw.), Vienna, Austria. Since September 2003, he has been with Infineon Technologies Austria AG. His research interests include multirate signal processing, filter banks, equalization, interference suppression, and channel and source coding and their application to telecommunications and image processing. 
Norbert J. Fliege received the Dipl.-Ing. degree and the Dr.-Ing. degree in 1971, both from the University of Karlsruhe, Germany. Since 1978, he was an Associate Professor at the same university. In 1980, he was a Visiting Professor at ESIEE in Paris. From 1982 to 1996, he was a Full Professor and Head of the Telecommunication Institute at Hamburg University of Technology, Ham-

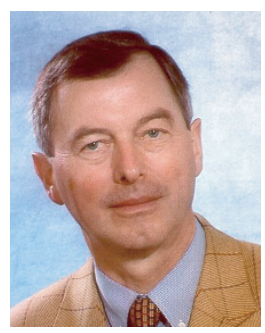
burg, Germany. Since 1996, he has been a

Full Professor of electrical engineering and computer technology at University of Mannheim, Germany. In 1997, he got the honorary doctorate from University of Rostock, Germany. Since 1968, Dr. Fliege has been engaged in research work on fields like active filters, digital filters, communication circuits and software, digital audio, and multirate digital signal processing. In addition, he served as a Department Chairman and as Head of a research center. He has also founded a company providing telecommunication equipment. Dr. Fliege has published about hundred papers, most of them in international magazines and conference proceedings, and four books, one of them with the title Multirate Digital Signal Processing, published by John Wiley and Sons in 1994. Dr. Fliege is a Fellow of IEEE, a Member of EURASIP, and a Member of VDE (Germany). 